\section{Interpretation of historical evidence}

SIR: Hare's paper on 'Schizophrenia as a recent disease' (Journal, October 1988, 153, 521-531) has attracted a considerable correspondence. Yet for such a theme, a much more detailed analysis is required than narrow research based on medical textbooks or annual statistics. Casting aspersions on Haslam's probity (Journal, September 1989, 155, 418-419) or forgetting that two leading London physicians actually believed James Tilly Matthews not to be insane (Illustrations of Madness (Haslam, 1810 ), is now available in reprint, so readers can easily decide) is also not historically illuminating.

Historical evidence in this context must not be selective. Writings on mental illness flourished in the late 18th and early 19th century, for unknown reasons. To suggest that a new form of illness, schizophrenia of adolescent onset, arose at this time is akin to believing that 'germ theory' arose because germs were new (rather than because new microscopic techniques allowed them to be studied properly for the first time).

In 1813 Thomas Sutton outlined delirium tremens; in 1817 James Parkinson delineated the 'shaking palsy'. Are we to believe that these were also new diseases? Given the extraordinary changes in English society between 1780 and 1820 - a period of ten called the 'Industrial Revolution' in traditional textbooks and the shift from an agrarian to an urban lifestyle, is it not more sensible to highlight a new perspective rather than a new form of illness? There are similar changes in medical practice (e.g. Laennec's first use of the stethoscope) at this time, and even by the end of the century 'hebephrenia' was regarded as a rare disorder (Dawson, 1903).

The financial aspects of asylum care also need examination. To what extent did some Victorian 'medical men' rely on lunacy certification for a significant part of their income? Was it cheaper for parish guardians to keep disordered individuals in asylums - after 1867 there was a 4 shilling subsidy or workhouses? What was the cost of caring for an insane relative at home?

Major epidemiological theories in the history of psychiatry are too important to be confined to the clinical. The task of understanding becomes much more complex, but that is the joy of history. While there is no doubt a value in asserting the primacy of illness in generating an asylum population, we cannot ignore the multiplier effects of industrial working hours, the population explosion, economic incentives, and degeneration theory, to say nothing of evangelical concern and a broader appreciation of psychiatric diagnoses. Admission under legal certifi- cation was and is a social event, not an act of refined nosology. Dr Hare's honesty in describing his evidence as "flimsy" deserves respect, but the view of schizophrenia as a persistent, genetically-based disorder may have sounder historical roots.

T. H. TURNER

Department of Psychological Medicine

The Hackney Hospital

Homerton High Street

London E96BE

References

DAwson, W. R. (1903) A case of hebephrenia. The Journal of Mental Science, 49, 303.

HASLAM, J. (1810, 1988) Illustrations of Madness. London: G. Hayden; reprinted London: Routledge.

\section{Nicotine and Alzheimer's disease}

SIR: We were pleased to read in the recent letter by Grant et al (Journal, November 1989, 155, 716) that they found our results (Journal, June 1989, 154, 797800 ) interesting and warranting further evaluation.

We agree with the point raised in their letter: that for the reasons they specify, patients who are regular smokers may not respond in the same way as nonsmokers. However, as Dr Grant et al would agree, changes in density of nicotinic receptors in the brain following repeated smoking does not provide evidence on its own of a functional change.

Dr Grant et al argue that the "heterogeneity of the patient population" may have resulted in a failure to detect a positive effect of nicotine on our test of shortterm memory. However, heterogeneity of patient population on its own would not be a sufficient explanation of our results, since we found marked and specific improvement in attention and information processing, including working memory.

Therefore one would have to resort to a more convoluted explanation involving perhaps some combination of factors, including differences in test sensitivity and a mixture of smoking and non-smoking patients. Since our Section is dedicated to assessing the efficacy of pharmacological treatments for dementia of the Alzheimer type (DAT), we would be only too pleased to find effects of nicotine on tests of spatial short-term memory. However, recent literature on experimental animals for spatial memory supports our findings (Mundy \& Iwamato, 1988).

Furthermore, we have just completed a large study of 71 subjects or patients ( 36 smokers and 35 nonsmokers) in order to investigate this question of response to nicotine in smokers and non-smokers. Results of our larger study have replicated nearly completely those of our earlier, smaller one, and we 